\title{
Microwave to optical photon conversion via fully concentrated rare-earth-ion crystals
}

\author{
Jonathan R. Everts, ${ }^{1}$ Matthew C. Berrington, ${ }^{2}$ Rose L. Ahlefeldt, ${ }^{2}$ and Jevon J. Longdell ${ }^{1}$ \\ ${ }^{1}$ Dodd-Walls Centre for Photonic and Quantum Technologies, Department of Physics, University of Otago, Dunedin 9016, New Zealand \\ ${ }^{2}$ Centre for Quantum Computation and Communication Technology, Research School of Physics and Engineering, \\ The Australian National University, Canberra, Australian Capital Territory 0200, Australia
}

(Received 16 December 2018; published 21 June 2019)

\begin{abstract}
Most investigations of rare-earth ions in solids for quantum information have used crystals where the rare-earth ion is a dopant. Here, we analyze the conversion of quantum information from microwave photons to optical frequencies using crystals where the rare-earth ions, rather than being dopants, are part of the host crystal. These concentrated crystals are attractive for frequency conversion because of their large ion densities and small linewidths. We show that conversion with both high efficiency and large bandwidth is possible in these crystals. In fact, the collective coupling between the rare-earth ions and the optical and microwave cavities is large enough that the limitation on the bandwidth of the devices will instead be the spacing between magnon modes in the crystal.
\end{abstract}

DOI: 10.1103/PhysRevA.99.063830

\section{INTRODUCTION}

Over recent years, superconducting qubits have emerged as a leading qubit design for quantum information processing [1-3]. In these systems, quantum information can readily be coupled into and out of the qubits via microwave photons. However, this means that they need to be operated at temperatures in the millikelvin range to not be swamped by thermal noise. It also makes long distance communication problematic because transferring quantum information with microwave photons would require a cryogenically cooled channel. Transferring quantum information to optical frequencies using a microwave-to-optical up-converter would get around this problem and enable the use of existing optical network technology.

To be useful for quantum computing, a microwave-tooptical up-converter needs nearly unity conversion efficiency for single photons and no added noise. Furthermore, a bandwidth greater than the coherence time of a superconducting qubit $(25$ and $7 \mathrm{kHz}$ for $2 \mathrm{D}$ and $3 \mathrm{D}$ transmon quantum circuits, respectively [4]) is required for the qubit to remain coherent following the conversion process. Faster conversion than this limit is beneficial up to the point where the conversion time is comparable to the gate time ( 75 and $30 \mathrm{MHz}$ for $2 \mathrm{D}$ and 3D transmon quantum circuits, respectively [4]).

This operating regime requires a system with a strong interaction with both optical and microwave fields, and a very strong nonlinearity to perform the conversion. Several different approaches have been investigated to achieve this regime. Electro-optomechanical approaches [5-11] have achieved the highest efficiency so far of $47 \%$, but the conversion bandwidth was limited to $12 \mathrm{kHz}$ and there were 38 photons of added noise [12,13]. Conversely, electro-optic materials [14-18] have demonstrated a $2 \%$ conversion efficiency but with a much larger bandwidth, of $0.59 \mathrm{MHz}$ at $2 \mathrm{~K}$ [19].

Approaches using atoms [20-22] have also been proposed. Recently, using the strong microwave and optical transitions of a cloud of Rydberg atoms at microkelvin temperatures, a conversion efficiency of $0.3 \%$ was achieved with a $4-\mathrm{MHz}$ bandwidth [23]. Collective spin excitations within a ferromagnet (magnons) have also been suggested. These resonances interact strongly with microwave cavity fields because of the very high density of spins. Low-efficiency upconversion has been demonstrated in a ferromagnetic crystal of yttrium iron garnet (YIG), limited by the weak coupling of the magnons to the optical field [24-27].

Atomlike systems in solids have been investigated including defects in diamond $[28,29]$ and rare-earth doped solids [30-33]. In these latter systems, the nonlinearity is obtained by simultaneously operating close to the narrow paramagnetic resonance (at microwave frequencies) and electronic resonance (at optical frequencies) of the rare-earth dopant. If the chosen dopant is erbium, with an optical resonance near $1.5 \mu \mathrm{m}$, this approach has the additional advantage of converting to the low-loss fiber telecommunications band. Only a low efficiency has been demonstrated so far, of $10^{-5}$ in $0.001 \% \mathrm{Er}: \mathrm{Y}_{2} \mathrm{SiO}_{5}$ at $5 \mathrm{~K}$ with $\approx 1 \mathrm{MHz}$ bandwidth [33].

A high nonlinearity, and thus conversion efficiency, in these rare-earth systems requires a high rare-earth concentration and low optical and spin-transition linewidths [30] so the system can be operated as close to the resonances as possible. However, these two desires are in conflict in dilute rare-earth crystals. The rare-earth dopant causes strain in the lattice, and as the concentration increases this leads to inhomogeneous broadening of the spectral line [34]. Fully concentrated crystals, however, can also show narrow optical inhomogeneous lines along with high optical depth, because the disorder due to randomly distributed dopant ions is removed. For example, an inhomogeneous linewidth of $25 \mathrm{MHz}$ has been observed in $\mathrm{Eu}^{35} \mathrm{Cl}_{3} \cdot 6 \mathrm{H}_{2} \mathrm{O}$ [35], comparable to the narrowest linewidths seen in dilute crystals [36,37].

Fully concentrated crystals also offer another feature: At temperatures around $1 \mathrm{~K}$, they can display magnetic order. The same magnon modes used in ferromagnetic YIG for 


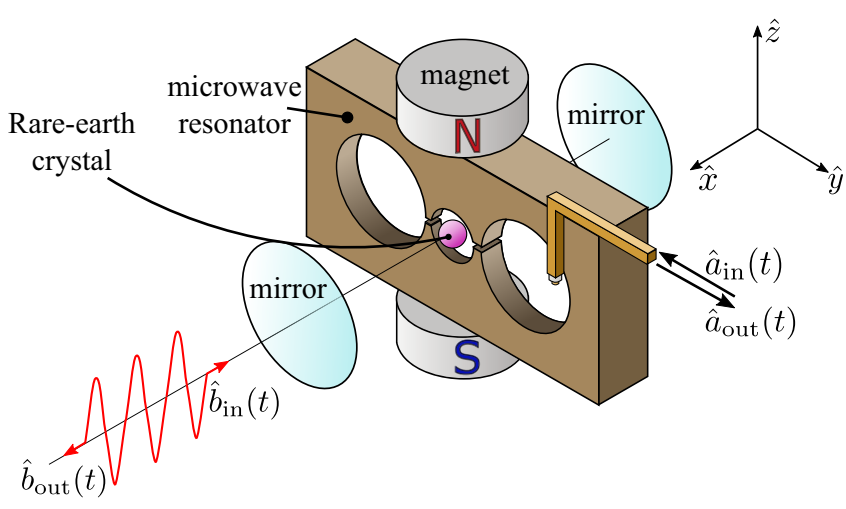

FIG. 1. The device used to convert microwave photons into optical photons. A rare-earth crystal (doped rare-earth crystal for previous device [30], fully concentrated for proposed device) is placed within a microwave resonator and an optical cavity. A static magnetic field is then applied in the $\hat{z}$ direction. This controls the frequency of the spin resonance (magnon resonance in current device).

upconversion are expected in pure rare-earth crystals, although the low ordering temperature means these have not been well studied. YIG does, however, provide a striking example that narrow lines are possible on these modes: the magnon resonance linewidth is as low as $0.6 \mathrm{MHz}[24,38]$, a result that first drove interest in using YIG for upconversion [39-41]. While the magnon lines in YIG are narrow, the available optical transitions come from iron and have inhomogeneous linewidths of hundreds of terahertz, which limits the achievable nonlinearity. This is not an issue in concentrated rare-earth crystals due to their narrow inhomogeneous lines.

In this paper, we propose and analyze a system for upconversion that combines the advantages of the ferromagnetic magnon and rare-earth approaches. We suggest using a magnetically ordered crystal fully concentrated in an optically active rare-earth ion. These systems are exciting for microwave to optical transduction because they promise very high atomic concentrations, but at the same time narrow optical transitions and narrow collective magnetic resonances.

\section{DEVICE OVERVIEW}

Our proposed device uses a similar process to a device proposed for low-concentration doped rare-earth crystals $[30,32,33]$, and we first briefly describe that device. The device is shown in Fig. 1. A doped rare-earth-ion crystal at cryogenic temperatures is coupled to a microwave resonator and an optical resonator. The dynamics of the device can be described by an off-resonant Raman-like process using the three-level energy diagram shown in Fig. 2(a). The rare-earth ions begin in their ground state $|g\rangle$, a microwave photon (frequency $\omega_{\mu}$ ) input into the microwave cavity excites a spin excitation within the doped rare-earth crystal, state $|1\rangle$. A coherent driving field resonant with one of the optical cavity modes (frequency $\omega_{\Omega}$ ) then excites an optical transition, state $|2\rangle$, where the rare-earth ion then returns to the ground state, emitting an optical photon resonant with another mode of the optical cavity (frequency $\omega_{o}$ ). All three fields are detuned from their respective transition resonances, but they are kept
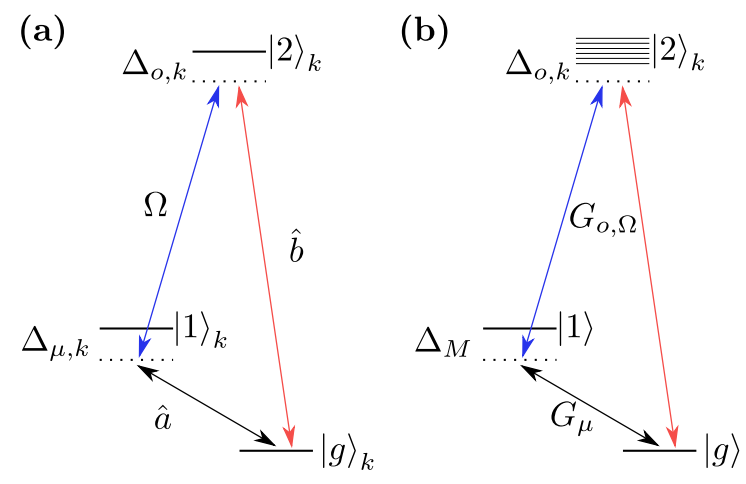

FIG. 2. (a) Energy-level diagram used to describe the dynamics of the previous conversion device [30]. An input microwave photon, in mode $\hat{a}$, excites a spin excitation in the $k$ th rare-earth ion, state $|1\rangle_{k}$. A coherent driving field with Rabi frequency $\Omega$ then drives the spin excitation to an optical excitation, state $|2\rangle_{k}$. From state $|2\rangle_{k}$, the device decays into the ground state $|g\rangle_{k}$, releasing an optical photon in mode $\hat{b}$ in the process. Each state transition is driven off resonantly, indicated by the detunings $\Delta_{o, k}$ and $\Delta_{\mu, k}$. (b) Energy-level diagram used to describe the dynamics of our proposed conversion device. Strong interactions between the spins means that using the single atom picture becomes problematic. The conversion occurs via a similar off-resonant Raman-like process to that of the previous conversion device. The state $|1\rangle$ has one excitation in the Kittel mode and is driven off resonance (an amount $\Delta_{M}$ ) by both a microwave input field (coupling strength $G_{\mu}$ ) and a two optical photon Raman process (coupling strength $G_{o, \Omega}$ ). Each optical state $|2\rangle_{k}$ is shifted due to the spin interactions. However, as the optical detuning $\Delta_{o, k}$ is much larger than this energy shift, the effect is negligible to our calculations.

in three-photon resonance, $\omega_{\mu}+\omega_{\Omega}=\omega_{o}$. Driving all transitions off resonantly greatly simplifies the dynamics of the device, because it allows us to adiabatically eliminate the dynamics of the excited levels.

Our proposed device works in the same manner as the device just described except that the device uses a fully concentrated rare-earth ion crystal rather than a doped crystal. In a high concentration regime, the strong interactions between rare-earth ions means the dynamics are best understood in collective excitations, rather than the individual ion energy states of the previous device. Similar to the previous device, the dynamics of the proposed device can be described by an off-resonant Raman-like process using the three-level energy diagram shown in Fig. 2(b); however, now state $|1\rangle$ is a single collective spin excitation (a magnon) that is excited both by direct microwave driving and by a two-optical-photon Raman process. Using just three levels, as shown in Fig. 2(a), is in distinct contrast to some approaches based on free atoms $[22,23]$ where selection rules lead to the use of extra levels and extra coupling fields. The lack of strong selection rules in low-symmetry rare-earth crystals allows $\Delta$ systems like this to be formed. These $\Delta$ systems are increasingly used to probe electron spin structure optically using Raman heterodyne spectroscopy [32,33,42-45].

In our device, we will be working in the regime where the microwave field wavelength is much greater than the rare-earth-ion crystal sample size. Under this condition, the 
magnon modes that can be excited within the sample do not propagate, and are known as magnetostatic modes. These modes are well understood in an isotropic spherical material [46-48]. The description of our proposed conversion device assumes that an input microwave photon excites only the lowest order, spatially uniform $(1,1,0)$ magnon mode or Kittel mode. This assumption is justified by operating with the other magnon modes highly detuned from both the microwave driving field and the driving by the two optical fields. Furthermore, choosing a microwave resonator with a uniform mode across the sample will mean that the other magnetostatic modes are only weakly driven, since those modes are spatially nonuniform.

We derive the device Hamiltonian in the Appendix, and show that it can be reduced to detunings and a coupling term between the microwave $(\hat{a})$ and optical $(\hat{b})$ modes,

$$
\mathcal{H}=\hbar \delta_{\mu} \hat{a}^{\dagger} \hat{a}+\hbar \delta_{o} \hat{b}^{\dagger} \hat{b}+\hbar\left(\xi \hat{a}^{\dagger} \hat{b}+\xi^{*} \hat{b}^{\dagger} \hat{a}\right)
$$

where $\delta_{\mu}, \delta_{o}$ are detunings of the microwave and optical fields from their respective cavities. The coupling strength, $\xi$, is given by

$$
\xi=\frac{G_{\mu}^{*} G_{o, \Omega}}{\Delta_{M}}
$$

with $\Delta_{M}$ the microwave detuning from the Kittel mode. The strengths of the Kittel mode coupling via the microwave input field $\left(G_{\mu}\right)$ and the two-optical-photon Raman process $\left(G_{o, \Omega}\right)$ are derived in the Appendix to be

$$
\begin{aligned}
G_{\mu} & =\frac{1}{\sqrt{N}} \sum_{k} g_{\mu, k}, \\
G_{o, \Omega} & =\frac{1}{\sqrt{N}} \sum_{k} \frac{\Omega_{k}^{*} g_{o, k}}{\Delta_{o, k}} .
\end{aligned}
$$

Here, the sum runs over all $N$ rare-earth ions in the sample, $g_{\mu, k}$ and $g_{o, k}$ represent the coupling strength between the $k$ th ion and the microwave and optical cavities, respectively, $\Omega_{k}$ is the Rabi frequency of the classical driving field and $\Delta_{o, k}$ is the detuning of the optical field from the $k$ th ion's optical transition.

\section{DEVICE EFFICIENCY}

In this section, the input-output formalism developed in Ref. [49] is used to calculate the relations between the microwave and optical cavity fields and their respective input/output modes. This allows us to define an impedance matching condition for the device, which we re-express in terms of interpretable parameters.

Applying the equation of motion for the field in a one-sided cavity, given by Eq. (6) and Eq. (19) in Ref. [49], the two cavity fields in the device evolve as

$$
\begin{aligned}
& \dot{a}(t)=-i \delta_{\mu} \hat{a}-i \xi \hat{b}(t)-\frac{\kappa_{\mu}}{2} \hat{a}(t)+\sqrt{\kappa_{\mu}} \hat{a}_{\mathrm{in}}(t), \\
& \dot{b}(t)=-i \delta_{o} \hat{b}-i \xi^{*} \hat{a}(t)-\frac{\kappa_{o}}{2} \hat{b}(t)+\sqrt{\kappa_{o}} \hat{b}_{\mathrm{in}}(t),
\end{aligned}
$$

where $\kappa_{\mu}$ and $\kappa_{o}$ represent the decay rates of the two cavities. Applying Eq. (5) in Ref. [49] also gives

$$
\begin{aligned}
& \dot{a}_{\text {out }}(t)+\dot{a}_{\text {in }}(t)=\sqrt{\kappa_{\mu}} \dot{a}(t), \\
& \dot{b}_{\text {out }}(t)+\dot{b}_{\text {in }}(t)=\sqrt{\kappa_{o}} \dot{b}(t) .
\end{aligned}
$$

Fourier transforming Eq. (5) and using Eq. (6) gives

$$
\begin{aligned}
\hat{a}_{\text {out }}(\omega=0)= & -\frac{|\xi|^{2}-\left(\frac{\kappa_{o}}{2}+i \delta_{o}\right)\left(\frac{\kappa_{\mu}}{2}-i \delta_{\mu}\right)}{|\xi|^{2}+\left(\frac{\kappa_{o}}{2}+i \delta_{o}\right)\left(\frac{\kappa_{\mu}}{2}+i \delta_{\mu}\right)} \hat{a}_{\text {in }} \\
& -\frac{i \xi \sqrt{\kappa_{o} \kappa_{\mu}}}{|\xi|^{2}+\left(\frac{\kappa_{o}}{2}+i \delta_{o}\right)\left(\frac{\kappa_{\mu}}{2}+i \delta_{\mu}\right)} \hat{b}_{\text {in }}, \\
\hat{b}_{\text {out }}(\omega=0)= & -\frac{|\xi|^{2}-\left(\frac{\kappa_{o}}{2}-i \delta_{o}\right)\left(\frac{\kappa_{\mu}}{2}+i \delta_{\mu}\right)}{|\xi|^{2}+\left(\frac{\kappa_{o}}{2}+i \delta_{o}\right)\left(\frac{\kappa_{\mu}}{2}+i \delta_{\mu}\right)} \hat{b}_{\text {in }} \\
& -\frac{i \xi^{*} \sqrt{\kappa_{o} \kappa_{\mu}}}{|\xi|^{2}+\left(\frac{\kappa_{o}}{2}+i \delta_{o}\right)\left(\frac{\kappa_{\mu}}{2}+i \delta_{\mu}\right)} \hat{a}_{\text {in }},
\end{aligned}
$$

where the Fourier time-conjugate $\omega$ was set to zero to describe steady-state dynamics. For both Eqs. (7) and (8), the first terms on the right side describe the signals reflected from the cavities. The second terms give the photon conversion between the microwave and optical fields. From these expressions, the number conversion efficiency is

$$
\eta=\left|\frac{\xi \sqrt{\kappa_{\mu} \kappa_{o}}}{|\xi|^{2}+\left(\frac{\kappa_{o}}{2}+i \delta_{o}\right)\left(\frac{\kappa_{\mu}}{2}+i \delta_{\mu}\right)}\right|^{2} .
$$

Provided the microwave and optical fields are resonant with the cavities $\left(\delta_{o} \ll \kappa_{o} / 2, \delta_{\mu} \ll \kappa_{\mu} / 2\right)$ and the strength of the coupling between the microwave and optical fields is chosen appropriately to achieve impedance matching $(2|\xi|=$ $\sqrt{\kappa_{\mu} \kappa_{o}}$ ), an input microwave field is completely converted into an output optical field and vice versa. The impedance matching condition can also be written as

$$
1=\frac{2\left|G_{\mu}\right|\left|G_{o, \Omega}\right|}{\kappa \Delta_{M}}
$$

The bandwidth of the device, meanwhile, is determined by the smaller of $\kappa_{o}$ and $\kappa_{\mu}$. For our feasibility analysis, later we will assume that they are equal to $\kappa_{o}=\kappa_{\mu}=\kappa$, giving a conversion bandwidth of $\sqrt{2} \kappa$.

To investigate if the impedance condition can realistically be achieved, we derive simplified expressions for $G_{\mu}, G_{o, \Omega}$. Setting the dipole moments $\boldsymbol{\mu}_{g 1, k}, \boldsymbol{d}_{g 2, k}, \boldsymbol{d}_{12, k}$, introduced in Eq. (A4), as homogeneous across the sample (independent of $k$ ), we can then take their scalar projections along the microwave and optical mode functions $\chi(\boldsymbol{r}), \boldsymbol{\phi}(\boldsymbol{r})$ and $\boldsymbol{\epsilon}(\boldsymbol{r})$, introduced in Eqs. (A5)-(A7). Because the optical detuning is much larger than the optical inhomogeneous linewidth, we also take $\Delta_{o, k}$ to be the same for each atom. Thus our expressions for $G_{\mu}$ and $G_{o, \Omega}$ become

$$
\begin{gathered}
G_{\mu}=\frac{1}{\sqrt{N}} \sqrt{\frac{\omega_{\mu} \mu_{0}}{2 \hbar V_{\mu}}} \mu_{g 1} \sum_{k=1}^{N}\left|\boldsymbol{\chi}\left(\boldsymbol{r}_{k}\right)\right|, \\
G_{o, \Omega}=\frac{1}{\sqrt{N}} \sqrt{\frac{\omega_{o}}{2 \hbar \epsilon_{0} V_{o}}} \frac{d_{g 2} \Omega_{0}}{\Delta_{o}} \sum_{k=1}^{N}\left|\boldsymbol{\phi}\left(\boldsymbol{r}_{k}\right)\right|\left|\boldsymbol{\epsilon}\left(\boldsymbol{r}_{k}\right)\right| .
\end{gathered}
$$


Approximating the sums as integrals over the crystal volume $\left(V_{c}\right)$ with atomic density $\rho=N / V_{c}$, we get

$$
\begin{gathered}
G_{\mu}=\sqrt{N} \sqrt{\frac{\omega_{\mu} \mu_{0}}{2 \hbar V_{\mu}}} \mu_{g 1} \frac{1}{V_{c}} \int_{V_{c}} d^{3} r\left|\chi\left(\boldsymbol{r}_{k}\right)\right| \\
=\sqrt{\rho V_{c}} \sqrt{\frac{\omega_{\mu} \mu_{0}}{2 \hbar V_{\mu}}} \mu_{g 1}, \\
G_{o, \Omega}=\sqrt{N} \sqrt{\frac{\omega_{o}}{2 \hbar \epsilon_{0} V_{o}}} \frac{d_{g 2} \Omega_{0}}{\Delta_{o}} \frac{1}{V_{c}} \int_{V_{c}} d^{3} r\left|\boldsymbol{\phi}\left(\boldsymbol{r}_{k}\right)\right|\left|\boldsymbol{\epsilon}\left(\boldsymbol{r}_{k}\right)\right| \\
=\sqrt{\rho V_{c}} \sqrt{\frac{\omega_{o}}{2 \hbar \epsilon_{0} V_{o}}} \frac{d_{g 2} \Omega_{0}}{\Delta_{o}} F .
\end{gathered}
$$

where we have defined $F$ as the integral over the optical fields divided by $V_{c}$, and the integral over the microwave field is simply $V_{c}$ because we assumed the field was uniform over the sample in Sec. II.

\section{FEASIBILITY}

In this section, we show that achieving unit efficiency is feasible in the device we propose using existing materials and reasonable experimental conditions. We assume the conversion operates between a 5-GHz-frequency microwave photon (similar to the frequency used for superconducting qubits), and an optical photon in the $1550-\mathrm{nm}$ telecommunications band. We consider a 2-mm diameter $\mathrm{ErCl}_{3} \cdot 6 \mathrm{H}_{2} \mathrm{O}$ sphere, a loop-gap microwave resonator, and Fabry Pérot optical resonator.

The rare-earth crystal that we consider is erbium chloride hexahydrate $\left(\mathrm{ErCl}_{3} \cdot 6 \mathrm{H}_{2} \mathrm{O}\right)$, a crystal that orders ferromagnetically below $350 \mathrm{mK}$ [50]. The optical transition of Er between the ${ }^{4} \mathrm{I}_{15 / 2}$ ground state and the ${ }^{4} \mathrm{I}_{13 / 2}$ excited state occurs at $1540 \mathrm{~nm}$. Using the maximum available $g$-factor of 13.1, an applied field of $\approx 30 \mathrm{mT}$ would bring the frequency of the Kittel mode to $5 \mathrm{GHz}$.

The optical and microwave cavities were modeled to determine the experimental parameters possible. The models are shown in Fig. 3. The microwave cavity is a shielded loopgap resonator, which produces a highly uniform magnetic field in the center of the cavity. We used finite-difference time-domain solutions to model the loop-gap resonator for a $5 \mathrm{GHz}$ resonance. The optical cavity was a Fabry-Pérot resonator with curved mirrors and had a $27-\mu \mathrm{m}$ waist inside the middle of the spherical sample. We use ray tracing with the paraxial approximation to model a Fabry Pérot resonator with a $1540-\mathrm{nm}$ resonance and $5-\mathrm{GHz}$ free spectral range. The $5-\mathrm{GHz}$ free spectral range was chosen so both optical fields are resonant with the cavity.

To justify the feasibility of the conversion device, we need to show four things: (a) that the impedance matching condition, Eq. (10), can be satisfied with physically realistic values; (b) that the Rabi frequency and optical cavity couplings are small compared to the optical detuning $\left(\Omega_{0}, g_{o} \ll \Delta_{o}\right)$; (c) that the cavity coupling to the magnon mode is small compared to the magnon detuning $\left(G_{\mu}, G_{o, \Omega} \ll \Delta_{M}\right)$; and (d) that the optical transition linewidth is small compared to the optical detuning $\left(\gamma_{o} \ll \Delta_{o}\right)$. While satisfying these four criteria will guarantee unit conversion efficiency, we also want (a) Loop-gap resonator

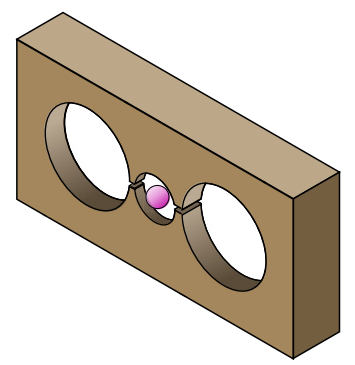

(b) Fabry-Pérot resonator

(c)
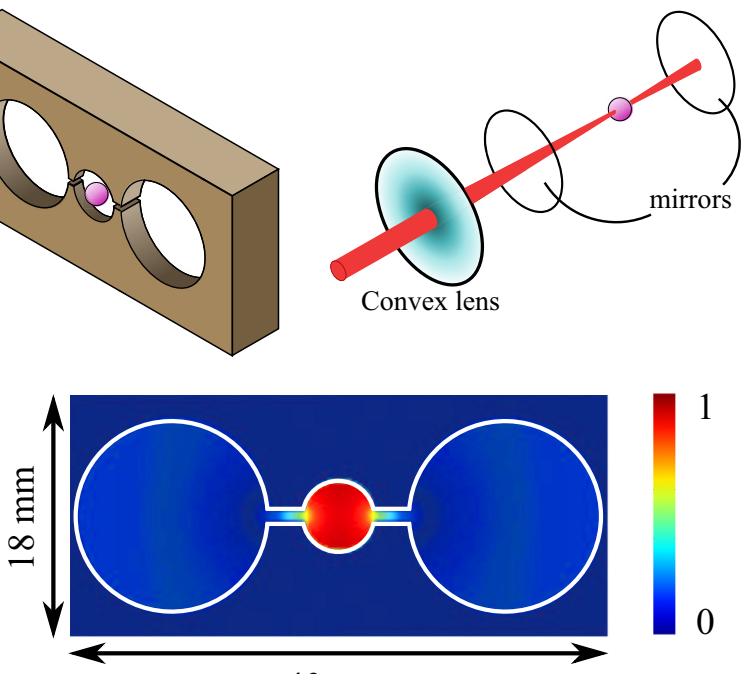

$40 \mathrm{~mm}$

(d)

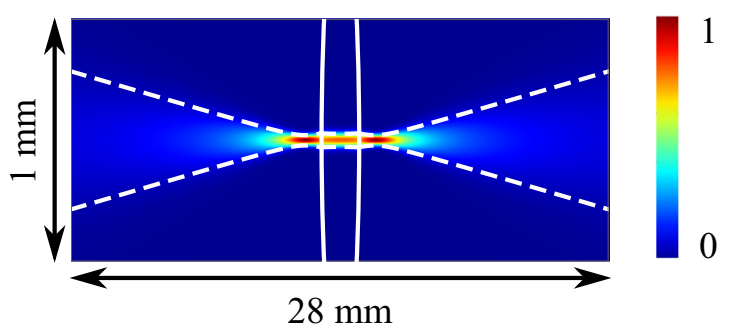

FIG. 3. Diagram showing proposed geometry (not to scale) for our (a) microwave resonator and (b) optical resonator. A convex lens is used in the optical resonator to focus the beam into the center of the sphere to minimize spherical aberration. (c), (d) show the modeled field amplitudes of the microwave and optical resonators. The solid white lines in (c) outline the loop gap, and in (d) outline the crystal of diameter $2 \mathrm{~mm}$. The dashed line in (d) indicates the Gaussian beam width.

to maximize the device conversion bandwidth, $\sqrt{2} \kappa$. This introduces a fifth design criteria (e) to maximize the device bandwidth $\sqrt{2} \kappa \propto G_{\mu} G_{o, \Omega}$ [see Eq. (10)] when possible.

We first consider satisfying the off-resonant condition (d). From Table I, the optical linewidth is $\gamma_{o}=1.24 \mathrm{GHz}$. By enforcing that the optical detuning is five times larger than the linewidth, $\Delta_{o}=6.2 \mathrm{GHz}$, we satisfy condition (d).

We now consider adiabatic condition (b). From Table I, the maximum Rabi frequency possible for the proposed device is $\Omega_{0}=68 \mathrm{MHz}$, assuming a maximum pump laser power of $1 \mu \mathrm{W}$. This is much less than the $6.2 \mathrm{GHz}$ optical detuning, so $\Omega_{0} \ll \Delta_{o}$. From the parameters in Table I, the coupling between the optical cavity and individual ions $g_{o}$, given by Eq. (A10), is between 0 and $1.5 \mathrm{kHz}$, depending on the direction of the optical field relative to the anisotropic transition dipole moment $d_{g 2}$. Thus $g_{o} \ll \Delta_{o}$, so condition (b) is satisfied.

We now consider adiabatic condition (c) and design parameter (e). From the parameters given in Table I, the coupling strength between the microwave cavity and the magnon mode $G_{\mu}$, given by Eq. (13), is between 0 and $1.6 \mathrm{GHz}$, 
TABLE I. Table of parameters for the proposed device for a 2-mm-diameter $\mathrm{ErCl}_{3} \cdot 6 \mathrm{H}_{2} \mathrm{O}$ crystal, loop-gap microwave resonator, and Fabry Pérot optical resonator. When the parameter can take on a range of values, the relevant extremum is given.

\begin{tabular}{lrr}
\hline \hline Parameter & Symbol & Value \\
\hline Crystal volume & $V_{c}$ & $4.2 \times 10^{-9} \mathrm{~m}^{3}$ \\
Atomic number density & $\rho$ & $4.0 \times 10^{27} \mathrm{~m}^{-3}[51]$ \\
Microwave angular frequency & $2 \pi \times 5 \mathrm{GHz}$ \\
Optical angular frequency & $\omega_{\mu}$ & $2 \pi \times 195 \mathrm{THz}$ \\
Maximum available magnetic dipole moment, $|g\rangle \rightarrow|1\rangle$ & $\omega_{o}$ & $1.5 \times 10^{-23} \mathrm{~J} \mathrm{~T}^{-1}[52]$ \\
Optical transition strength, $|g\rangle \rightarrow|2\rangle$ & $\max \left(\mu_{g 1}\right)$ & $2.0 \times 10^{-32} \mathrm{Cm}^{\mathrm{a}}$ \\
Optical transition FWHM & $d_{g 2}$ & $1.24 \mathrm{GHz}^{\mathrm{b}}$ \\
Microwave cavity mode volume & $\gamma_{o}$ & $2.9 \times 10^{-7} \mathrm{~m}^{3} \mathrm{c}$ \\
Optical cavity mode volume & $V_{\mu}$ & $2.9 \times 10^{-11} \mathrm{~m}^{3} \mathrm{~d}$ \\
Optical field overlap integral & $V_{o}$ & $2.4 \times 10^{-4 \mathrm{~d}}$ \\
Maximum available Rabi frequency & $F$ & $68 \mathrm{MHz}^{\mathrm{e}}$ \\
Maximum available microwave cavity quality factor & $9 \times 10^{4 \mathrm{f}}$ \\
Maximum available optical cavity quality factor & $\max \left(\Omega_{0}\right)$ & $9 \times 1.2 \times 10^{8 \mathrm{~g}}$ \\
\hline \hline
\end{tabular}

a Measured from absorption experiments on $\mathrm{Er}: \mathrm{EuCl}_{3} \cdot 6 \mathrm{H}_{2} \mathrm{O}$, which is isostructural with $\mathrm{ErCl}_{3} \cdot 6 \mathrm{H}_{2} \mathrm{O} \cdot 6 \mathrm{H}_{2} \mathrm{O}$. Given here as an effective electric dipole moment but the transition likely has a significant magnetic component. This is not uncommon for Er dopants [53].

${ }^{\mathrm{b}}$ Measured from absorption spectroscopy experiments performed on $\mathrm{ErCl}_{3} \cdot 6 \mathrm{H}_{2} \mathrm{O}$ at $5 \mathrm{~K}$ and $3.5 \mathrm{~T}$, unpublished.

${ }^{c}$ Obtained using finite-difference time-domain solutions for the loop gap resonator.

${ }^{\mathrm{d}}$ Obtained from a numerical paraxial model of the Fabry Pérot resonator.

${ }^{e}$ Calculated assuming a maximum pump laser power of $1 \mu \mathrm{W}$, a typical tolerable load for cryogenic systems. The beam width and cavity field enhancement was calculated using the Fabry Pérot resonator model. See Supplemental Material [54].

${ }^{\mathrm{f}}$ Easily achieved with 3D copper microwave resonators [32].

'The quality factor attainable for a Fabry Pérot cavity with optimized output coupling [33].

depending on the direction of the microwave field relative to the anisotropic transition dipole moment $\mu_{g 1}$. Meanwhile, the coupling between the optical cavity and the magnon mode $G_{o, \Omega}$ [Eq. (14)] is between 0 and $63 \mathrm{MHz}$, depending on the choice of the Rabi frequency $\Omega_{0}$. The largest feasible detuning from the Kittel mode $\Delta_{M}$, is given by the frequency spacing to the next magnon mode. At microwave frequencies of $5 \mathrm{GHz}$, this spacing is expected to be $\mathcal{O}(100 \mathrm{MHz})$ [47]. Given the tunability of $G_{\mu}, G_{o, \Omega}$, we can satisfy (e) by maximizing them within the constraint of condition (c). This can be achieved by selecting $G_{\mu}=G_{o, \Omega}=10 \mathrm{MHz}$ and $\Delta_{M}=100 \mathrm{MHz}$, where the factor of 10 difference guarantees $G_{\mu}, G_{o, \Omega} \ll \Delta_{M}$.

Finally, we consider the impedance matching condition (a). To satisfy Eq. (10) with the values of $G_{\mu}, G_{o, \omega}, \Delta_{M}$ chosen above requires the cavity decay rates to be $\kappa=2 \mathrm{MHz}$. This necessitates the quality factors of the microwave and optical cavities to be $Q_{\mu}=2.5 \times 10^{3}$ and $Q_{o}=9.7 \times 10^{7}$, respectively. From Table I, this is within the range of feasible quality factors, so satisfying (a) is physically achievable.

This means our proposed device can satisfy all the requirements of the theory set out in the Appendix and Sec. III with bandwidths well in excess of one megahertz possible. We note that, when choosing parameters, the limiting factor on the bandwidth was the spacing between the magnon modes: Tthe bandwidth available is set by the collective coupling rates which were in turn limited by the adiabatic condition to be less than the magnon detuning, which is in turn limited by the spacing between the magnon modes. This means that the reliability of this feasibility analysis is somewhat insensitive to all the other system parameters. It is unfortunate that of all the parameters we have estimated, the spacing between the magnon modes is the least well constrained, in particular the calculations in Ref. [47] are for an isotropic ferromag- net, which $\mathrm{ErCl}_{3} \cdot 6 \mathrm{H}_{2} \mathrm{O}$ is not. For the optical properties of $\mathrm{ErCl}_{3} \cdot 6 \mathrm{H}_{2} \mathrm{O}$ and the Zeeman sensitivity of the spin states, we used measured quantities, but collective magnetic resonance is poorly studied in crystals with rare-earth ions as the only magnetic ion. The only observation we are aware of is Ref. [55]. This is perhaps because rare-earth ions have weak exchange interactions and only order at temperatures comparable to $1 \mathrm{~K}$. Additionally, narrow collective resonances are only seen at temperatures well below the Curie temperature [56] and this requirement for very low temperatures would rule such materials out for many traditional applications of magnetic materials. This is not a problem when using these materials for frequency conversion of microwave photons because microwave-frequency quantum states already require these very low temperatures.

\section{CONCLUSION}

We proposed using insulating crystals fully concentrated with rare-earth ions for microwave-to-optical frequency conversion. These materials promise very narrow optical transitions at the same time as very high ion densities and narrow collective magnetic resonances. The analysis presented showed that using $\mathrm{ErCl}_{3} \cdot 6 \mathrm{H}_{2} \mathrm{O}$ as the crystal, high-efficiency, high-bandwidth microwave-to-optical conversion should be possible. This result suggests further study of fully concentrated rare-earth crystals, particularly their collective resonances, would be a promising avenue.

\section{ACKNOWLEDGMENTS}

The authors would like to thank M. Sellars for useful discussions. J.R.E. and J.J.L. were supported by the Marsden 
Fund of the Royal Society of New Zealand through Contract No. UOO1520. R.L.A. is a recipient of an Australian Research Council Discovery Early Career Researcher Award (Project No. DE170100099) funded by the Australian Government.

\section{APPENDIX: DEVICE HAMILTONIAN}

The Hamiltonian for our device can be written as

$$
\mathcal{H}=H_{\mathrm{F}}+H_{\mathrm{A}}+H_{\mathrm{IF}}+H_{\mathrm{E}}+H_{\mathrm{D}}
$$

Here, $H_{\mathrm{F}}$ describes the energy in the cavity fields,

$$
H_{\mathrm{F}}=\hbar \omega_{\mu, c} \hat{a}^{\dagger} \hat{a}+\hbar \omega_{o, c} \hat{b}^{\dagger} \hat{b},
$$

where $\hat{a}$ and $\hat{b}$ are the annihilation operators of the microwave and optical cavities, respectively, and $\omega_{\mu, c}$ and $\omega_{o, c}$ are the (bare) resonant frequencies of the microwave and optical cavities, respectively.

$H_{\mathrm{A}}$ describes the energy of the rare-earth ions:

$$
H_{\mathrm{A}}=\sum_{k} \hbar \omega_{2, k} \sigma_{22, k}+\hbar \omega_{1, k} \sigma_{11, k} .
$$

The sum here is over all rare-earth ions within the sphere and the subscript $k$ indicates the $k$ th rare-earth ion. The resonant frequency of the excited states $|1\rangle_{k}$ and $|2\rangle_{k}$ are given by $\omega_{1, k}$ and $\omega_{2, k}$, respectively, and $\sigma_{i j} \equiv|i\rangle\langle j|$ represents the atomic transition operator. This term has taken into account the energy splitting due to the Zeeman interaction, such that $\hbar \omega_{1, k}=g \mu_{B} B_{0}$, where $g$ is the Landé $g$-factor, $\mu_{B}$ is the Bohr magneton, and $B_{0}$ is the strength of the static magnetic field.

$H_{\mathrm{IF}}$ describes the interaction between the rare-earth ions and the cavity fields:

$$
H_{\mathrm{IF}}=\sum_{k} \boldsymbol{\mu}_{g 1, k} \cdot \boldsymbol{B}_{\mu}\left(\boldsymbol{r}_{k}\right)+\boldsymbol{d}_{g 2, k} \cdot \boldsymbol{E}_{o}\left(\boldsymbol{r}_{k}\right)+\boldsymbol{d}_{12, k} \cdot \boldsymbol{E}_{\Omega}\left(\boldsymbol{r}_{k}, t\right) .
$$

Here, $\boldsymbol{\mu}_{g 1}$ is the magnetic dipole operator of the microwave transition and $\boldsymbol{d}_{12}$ and $\boldsymbol{d}_{g 2}$ are the electric dipole operators of the optical and driving field transitions, respectively. $\boldsymbol{B}_{\mu}, \boldsymbol{E}_{o}$, and $\boldsymbol{E}_{\Omega}$ describe the magnetic and electric field operators for the microwave, optical, and driving fields, respectively,

$$
\begin{gathered}
\boldsymbol{B}_{\mu}=\sqrt{\frac{\hbar \mu_{0} \omega_{\mu, c}}{2 V_{\mu}}}\left(\hat{a}^{\dagger}+\hat{a}\right) \chi\left(\boldsymbol{r}_{k}\right), \\
\boldsymbol{E}_{o}=\sqrt{\frac{\hbar \omega_{o, c}}{2 \epsilon_{0} V_{o}}}\left(\hat{b}^{\dagger}+\hat{b}\right) \boldsymbol{\phi}\left(\boldsymbol{r}_{k}\right), \\
\boldsymbol{E}_{\Omega}=E_{0}\left(e^{-i \omega_{\Omega} t}+e^{i \omega_{\Omega} t}\right) \boldsymbol{\epsilon}\left(\boldsymbol{r}_{k}\right),
\end{gathered}
$$

where the mode volumes of the microwave and optical cavities are represented by $V_{\mu}$ and $V_{o}$, respectively, and $E_{0}$ is the peak magnitude of the coherent driving field. The microwave and optical mode functions are represented by $\chi(\boldsymbol{r}), \boldsymbol{\phi}(\boldsymbol{r})$ and $\boldsymbol{\epsilon}(\boldsymbol{r})$, respectively, and have been normalized between 0 and 1 .
Expanding Eq. (A4) out, we can obtain the expression

$$
\begin{aligned}
H_{\mathrm{IF}}= & \sum_{k} \hbar g_{\mu, k}\left(\sigma_{g 1, k}+\sigma_{1 g, k}\right)\left(\hat{a}^{\dagger}+\hat{a}\right) \\
& +\hbar g_{o, k}\left(\sigma_{g 2, k}+\sigma_{2 g, k}\right)\left(\hat{b}^{\dagger}+\hat{b}\right) \\
& +\hbar \Omega_{k}\left(\sigma_{12, k}+\sigma_{21, k}\right)\left(e^{-i \omega_{\Omega} t}+e^{i \omega_{\Omega} t}\right) .
\end{aligned}
$$

Here the coupling strengths between the ions and the microwave and optical cavities are given by $g_{\mu}$ and $g_{o}$, respectively,

$$
\begin{aligned}
g_{\mu, k} & =\sqrt{\frac{\omega_{\mu, c} \mu_{0}}{2 \hbar V_{\mu}}} \boldsymbol{\mu}_{g 1, k} \cdot \boldsymbol{\chi}\left(\boldsymbol{r}_{k}\right), \\
g_{o, k} & =\sqrt{\frac{\omega_{o, c}}{2 \hbar \epsilon_{0} V_{o}}} \boldsymbol{d}_{g 2, k} \cdot \boldsymbol{\phi}\left(\boldsymbol{r}_{k}\right),
\end{aligned}
$$

and the Rabi frequency of the classical driving field for each ion is represented by $\Omega$,

$$
\begin{aligned}
\Omega_{k} & =\frac{1}{\hbar} \boldsymbol{d}_{12, k} \cdot E_{0} \boldsymbol{\epsilon}\left(\boldsymbol{r}_{k}\right) \\
& =\boldsymbol{\Omega}_{0, k} \cdot \boldsymbol{\epsilon}\left(\boldsymbol{r}_{k}\right),
\end{aligned}
$$

where $\boldsymbol{\Omega}_{0, k}$ is the peak Rabi frequency.

The final two terms describe interactions between the spins that generate collective behavior. $H_{\mathrm{E}}$ describes the exchange interaction between neighboring spins,

$$
H_{\mathrm{E}}=-J \sum_{k, \delta} \hat{\boldsymbol{S}}_{k} \cdot \hat{\boldsymbol{S}}_{k+\delta} .
$$

Here, the $\delta$ summation runs over all nearest neighbors to the $k$ th rare-earth ion and $J$ is the isotropic exchange constant.

Finally, $H_{\mathrm{D}}$ describes the dipole-dipole interaction between spins:

$$
H_{\mathrm{D}}=\frac{1}{2} g^{2} \mu_{B}^{2} \sum_{k, j}\left[\frac{\hat{\boldsymbol{S}}_{k} \cdot \hat{\boldsymbol{S}}_{j}}{r_{k j}^{3}}-\frac{3\left(\hat{\boldsymbol{S}}_{k} \cdot \boldsymbol{r}_{k j}\right)\left(\hat{\boldsymbol{S}}_{j} \cdot \boldsymbol{r}_{k j}\right)}{r_{k j}^{5}}\right] .
$$

Here, both the $k$ and $j$ summations run over all rare-earth ions, and $\boldsymbol{r}_{k j}$ is the displacement vector from the $k$ th rare-earth ion to the $j$ th rare-earth ion.

As we are working within a regime where only magnetostatic modes are excited, the effect of the exchange interaction is negligible and can be ignored $[46,47]$. Further, following the method of Holstein and Primakoff [57], it can be shown that for a spherical sample in which only the Kittel mode is excited, the dipole-dipole interaction reduces to a constant which can be ignored when analyzing the dynamics of the device. The reduction of the dipole-dipole interaction to a constant occurs due to the spherical shape of the sample, in which it is well known that an applied field causes a uniform magnetization. Each spin therefore sees the same self-field, which is the reason narrow linewidths are seen in uniform collective spin resonances.

Before moving our Hamiltonian into the interaction picture, we rewrite $H_{\mathrm{F}}$ and $H_{\mathrm{A}}$ in the following way:

$$
\begin{aligned}
H_{\mathrm{F}} & =\hbar \omega_{o} \hat{b}^{\dagger} \hat{b}+\hbar \omega_{\mu} \hat{a}^{\dagger} \hat{a}+\hbar\left(\omega_{\mu, c}-\omega_{\mu}\right) \hat{a}^{\dagger} \hat{a}+\hbar\left(\omega_{o, c}-\omega_{o}\right) \hat{b}^{\dagger} \hat{b} \\
& =\hbar \omega_{o} \hat{b}^{\dagger} \hat{b}+\hbar \omega_{\mu} \hat{a}^{\dagger} \hat{a}+\hbar \delta_{\mu} \hat{a}^{\dagger} \hat{a}+\hbar \delta_{o} \hat{b}^{\dagger} \hat{b}, \quad \text { (A14) }
\end{aligned}
$$




$$
\begin{aligned}
H_{\mathrm{A}}= & \sum_{k} \hbar \omega_{o} \sigma_{22, k}+\hbar \omega_{\mu} \sigma_{11, k} \\
& +\hbar\left(\omega_{2, k}-\omega_{o}\right) \sigma_{22, k}+\hbar\left(\omega_{1, k}-\omega_{\mu}\right) \sigma_{11, k} \\
= & \sum_{k} \hbar \omega_{o} \sigma_{22, k}+\hbar \omega_{\mu} \sigma_{11, k} \\
& +\hbar \Delta_{o, k} \sigma_{22, k}+\hbar \Delta_{\mu, k} \sigma_{11, k} .
\end{aligned}
$$

Here, $\omega_{o}$ and $\omega_{\mu}$ are the frequencies of the applied optical and microwave fields, respectively. The detunings of the microwave and optical fields from the cavities are $\delta_{\mu}$ and $\delta_{o}$, respectively, and the detuning of the microwave and optical fields from the spin and optical transitions are $\Delta_{\mu}$ and $\Delta_{o}$, respectively.

Writing our Hamiltonian as $\mathcal{H}=H_{0}+V$, where

$$
\begin{gathered}
H_{0}=\hbar \omega_{\mu} \hat{a}^{\dagger} \hat{a}+\hbar \omega_{o} \hat{b}^{\dagger} \hat{b} \\
+\sum_{k}\left(\hbar \omega_{o} \sigma_{22, k}+\hbar \omega_{\mu} \sigma_{11, k}\right), \\
V=\hbar \delta_{\mu} \hat{a}^{\dagger} \hat{a}+\hbar \delta_{o} \hat{b}^{\dagger} \hat{b} \\
+\sum_{k}\left(\hbar \Delta_{o, k} \sigma_{22, k}+\hbar \Delta_{\mu, k} \sigma_{11, k}\right)+H_{\mathrm{IF}},
\end{gathered}
$$

we move into the interaction picture via the transformation $\mathcal{H}=e^{i H_{0} t / \hbar} V e^{-i H_{0} t / \hbar}$ to get

$$
\begin{aligned}
\mathcal{H}= & \hbar \delta_{\mu} \hat{a}^{\dagger} \hat{a}+\hbar \delta_{o} \hat{b}^{\dagger} \hat{b}+\sum_{k}\left(\hbar \Delta_{o, k} \sigma_{22, k}+\hbar \Delta_{\mu, k} \sigma_{11, k}\right) \\
& +\sum_{k}\left(\Omega_{k} \sigma_{21, k}+\text { H.c. }\right)+\sum_{k}\left(g_{\mu, k} \sigma_{1 g, k} \hat{a}+\text { H.c. }\right) \\
& +\sum_{k}\left(g_{o, k} \sigma_{2 g, k} \hat{b}+\text { H.c. }\right) .
\end{aligned}
$$

We will now simplify our device Hamiltonian by removing the dynamics of the optical and Kittel modes, which will enable the conversion efficiency of our device to be calculated in Sec. III. This simplification is possible as our system is driven off-resonantly, thus the $|1\rangle$ and $|2\rangle$ states will remain nearly unpopulated, acting only as intermediate states to enable a three-photon process. Removing the dynamics of the intermediate states can be achieved by the process of adiabatic elimination; for details on the process see Refs. [58,59].

We first adiabatically eliminate the optically excited state from each atom. Working with large optical detunings, $\left|\Delta_{o, k}\right| \gg\left|g_{o, k}\right|$ and $\left|\Delta_{o, k}\right| \gg\left|\Omega_{k}\right|$, we can adiabatically eliminate the optically excited states from each atom to obtain

$$
\begin{aligned}
\mathcal{H}= & \hbar \delta_{\mu} \hat{a}^{\dagger} \hat{a}+\hbar \delta_{o} \hat{b}^{\dagger} \hat{b}+\hbar \sum_{k} \Delta_{\mu, k} \sigma_{11, k} \\
& -\hbar \sum_{k}\left(\frac{\left|\Omega_{k}\right|^{2}}{\Delta_{o, k}}\right) \sigma_{11, k} \\
& -\hbar \sum_{k} \frac{1}{\Delta_{o, k}}\left|g_{o, k}\right|^{2} \sigma_{g g, k} \hat{b}^{\dagger} \hat{b} \\
& +\hbar \sum_{k}\left(g_{\mu, k} \sigma_{1 g, k} \hat{a}+\text { H.c. }\right) \\
& -\hbar \sum_{k} \frac{1}{\Delta_{o, k}}\left(g_{o, k} \Omega_{k}^{*} \sigma_{1 g, k} \hat{b}+\text { H.c. }\right) .
\end{aligned}
$$

Three new terms appear in the Hamiltonian. The fourth term of Eq. (A19) describes the ac stark shift, which under the adiabatic elimination condition $\left(\left|\Delta_{o, k}\right| \gg\left|\Omega_{k}\right|\right)$ will be small and hence ignored. The fifth term in Eq. (A19) represents a shift in the resonance frequency of the optical cavity due to the presence of the atoms. This term can be compensated for by tuning the resonant frequency of the optical cavity. This can be seen from Eq. (A19) by rewriting, without loss of generality, the optical cavity detuning as $\delta_{o} \rightarrow \delta_{o}+\delta_{o}^{\prime}$ where $\delta_{o}^{\prime}=\sum_{k} \frac{1}{\Delta_{o, k}}\left|g_{o, k}\right|^{2} \sigma_{g g, k}$. The final term in Eq. (A19) describes the coupling between the ground and excited states via the two optical fields.

To adiabatically eliminate the first excited state, the Kittel mode, we need to express the Hamiltonian in terms of Kittel mode creation and annihilation operators. This transformation will be carried out in several steps. First, we can re-express the Hamiltonian in terms of spin operators, via the transformations

$$
\frac{1}{2}\left(\sigma_{g g, k}-\sigma_{11, k}\right) \rightarrow \hat{S}_{z, k}, \sigma_{g 1, k} \rightarrow \hat{S}_{+, k}, \sigma_{1 g, k} \rightarrow \hat{S}_{-, k} .
$$

Second, we introduce the Holstein-Primakoff transformations for effective spin-half systems [60],

$$
\begin{aligned}
\hat{S}_{+, k} & =\hat{h}_{k} \\
\hat{S}_{-, k} & =\hat{h}_{k}^{\dagger} \\
\hat{S}_{z, k} & =\left(\frac{1}{2}-\hat{h}_{k}^{\dagger} \hat{h}_{k}\right),
\end{aligned}
$$

where $\hat{h}$ are the Holstein-Primakoff operators.

Finally, we introduce a transformation between the Holstein-Primakoff operators and the Kittel mode operator. This transformation involves a Fourier transform followed by a Bogoliubov transformation [57]. However, for a spherical sample and by ignoring all modes other than the Kittel mode, these transformations reduce to

$$
\hat{h}_{k}=\frac{1}{\sqrt{N}} \hat{m}_{0},
$$

where $N$ is the number of rare-earth ions in the spherical crystal and $\hat{m}_{0}$ is the Kittel mode annihilation operator.

Applying transformations, Eqs. (A20), (A21), and (A22) to Eq. (A19), we obtain a Hamiltonian in terms of Kittel mode operators,

$$
\begin{aligned}
\mathcal{H}= & \hbar \delta_{\mu} \hat{a}^{\dagger} \hat{a}+\hbar \delta_{o} \hat{b}^{\dagger} \hat{b}+\hbar \Delta_{M} \hat{m}_{0}^{\dagger} \hat{m}_{0} \\
& +\left[\hbar \hat{m}_{0}\left(G_{\mu}^{*} \hat{a}^{\dagger}-G_{o, \Omega}^{*} \hat{b}^{\dagger}\right)+\text { H.c. }\right],
\end{aligned}
$$

where we have introduced the parameters

$$
\begin{aligned}
G_{\mu} & =\frac{1}{\sqrt{N}} \sum_{k} g_{\mu, k}, \\
G_{o, \Omega} & =\frac{1}{\sqrt{N}} \sum_{k} \frac{\Omega_{k}^{*} g_{o, k}}{\Delta_{o, k}}, \\
\Delta_{M} & =\frac{1}{N} \sum_{k} \Delta_{\mu, k} .
\end{aligned}
$$

These parameters have a physical interpretation: $G_{\mu}$ describes the coupling between the microwave cavity and the 
Kittel mode, $G_{o, \Omega}$ describes the coupling between the optical cavity and the Kittel mode via the two-step process $|g\rangle \rightarrow$ $|2\rangle \rightarrow|1\rangle$, and $\Delta_{M}$ describes the detuning of the microwave field from the Kittel mode transition.

Working with $G_{\mu}, G_{o, \Omega} \ll \Delta_{M}$, we can adiabatically eliminate the Kittel mode from Eq. (A23) to obtain

$$
\begin{aligned}
\mathcal{H}= & \hbar \delta_{\mu} \hat{a}^{\dagger} \hat{a}+\hbar \delta_{o} \hat{b}^{\dagger} \hat{b} \\
& -\frac{\hbar}{\Delta_{M}}\left|G_{\mu}\right|^{2} \hat{a}^{\dagger} \hat{a}-\frac{\hbar}{\Delta_{M}}\left|G_{o, \Omega}\right|^{2} \hat{b}^{\dagger} \hat{b} \\
& +\frac{\hbar}{\Delta_{M}}\left(G_{\mu}^{*} G_{o, \Omega} \hat{a}^{\dagger} \hat{b}+G_{\mu} G_{o, \Omega}^{*} \hat{b}^{\dagger} \hat{a}\right) .
\end{aligned}
$$

Similar to what we saw in Eq. (A19), the third and fourth terms of Eq. (A27) represent a shift in the resonance frequency of the two cavities due to the presence of the atoms. These terms can be compensated for in experiment by tuning the cavities (setting $\delta_{\mu} \rightarrow \delta_{\mu}+\delta_{\mu}^{\prime \prime}$, where $\delta_{\mu}^{\prime \prime}=\frac{1}{\Delta_{M}}\left|G_{\mu}\right|^{2}$ and $\delta_{o} \rightarrow$ $\delta_{o}+\delta_{o}^{\prime \prime}$, where $\left.\delta_{o}^{\prime \prime}=\frac{1}{\Delta_{M}}\left|G_{o, \Omega}\right|^{2}\right)$. The third term represents a linear coupling between the microwave and optical cavity fields, with strength $\xi$ :

$$
\xi=\frac{G_{\mu}^{*} G_{o, \Omega}}{\Delta_{M}} .
$$

We have now arrived at a simplified Hamiltonian for the device, which will be used to estimate the device performance:

$$
\mathcal{H}=\hbar \delta_{\mu} \hat{a}^{\dagger} \hat{a}+\hbar \delta_{o} \hat{b}^{\dagger} \hat{b}+\hbar\left(\xi \hat{a}^{\dagger} \hat{b}+\xi^{*} \hat{b}^{\dagger} \hat{a}\right) .
$$

[1] Y. Nakamura, Y. Pashkin, and J.-S. Tsai, Nature 398, 786 (1999).

[2] M. H. Devoret and R. J. Schoelkopf, Science 339, 1169 (2013).

[3] J. Q. You and F. Nori, Phys. Today 58(11), 42 (2005).

[4] G. Wendin, Rep. Prog. Phys. 80, 106001 (2017).

[5] S. Barzanjeh, M. Abdi, G. J. Milburn, P. Tombesi, and D. Vitali, Phys. Rev. Lett. 109, 130503 (2012).

[6] Y.-D. Wang and A. A. Clerk, Phys. Rev. Lett. 108, 153603 (2012).

[7] J. Bochmann, A. Vainsencher, D. D. Awschalom, and A. N. Cleland, Nat. Phys. 9, 712 (2013).

[8] T. Bagci, A. Simonsen, S. Schmid, L. G. Villanueva, E. Zeuthen, J. Appel, J. M. Taylor, A. Sørensen, K. Usami, A. Schliesser, and E. S. Polzik, Nature 507, 81 (2014).

[9] K. Zhang, F. Bariani, Y. Dong, W. Zhang, and P. Meystre, Phys. Rev. Lett. 114, 113601 (2015).

[10] F. Lecocq, J. B. Clark, R. W. Simmonds, J. Aumentado, and J. D. Teufel, Phys. Rev. Lett. 116, 043601 (2016).

[11] C. F. Ockeloen-Korppi, E. Damskägg, J.-M. Pirkkalainen, T. T. Heikkilä, F. Massel, and M. A. Sillanpää, Phys. Rev. X 6, 041024 (2016).

[12] R. W. Andrews, R. W. Peterson, T. P. Purdy, K. Cicak, R. W. Simmonds, C. A. Regal, and K. W. Lehnert, Nat. Phys. 10, 321 (2014).

[13] A. P. Higginbotham, P. S. Burns, M. D. Urmey, R. W. Peterson, N. S. Kampel, B. M. Brubaker, G. Smith, K. W. Lehnert, and C. A. Regal, Nat. Phys. 14, 1038 (2018).

[14] M. Tsang, Phys. Rev. A 81, 063837 (2010).

[15] M. Tsang, Phys. Rev. A 84, 043845 (2011).

[16] A. Rueda, F. Sedlmeir, M. C. Collodo, U. Vogl, B. Stiller, G. Schunk, D. V. Strekalov, C. Marquardt, J. M. Fink, O. Painter, G. Leuchs, and H. G. L. Schwefel, Optica 3, 597 (2016).

[17] J. D. Witmer, J. A. Valery, P. Arrangoiz-Arriola, C. J. Sarabalis, J. T. Hill, and A. H. Safavi-Naein, Sci. Rep. 7, 46313 (2017).

[18] M. Soltani, M. Zhang, C. Ryan, G. J. Ribeill, C. Wang, and M. Loncar, Phys. Rev. A 96, 043808 (2017).

[19] L. Fan, C.-L. Zou, R. Cheng, X. Guo, X. Han, Z. Gong, S. Wang, and H. X. Tang, Sci. Adv. 4, eaar4994 (2018).

[20] B. T. Gard, K. Jacobs, R. McDermott, and M. Saffman, Phys. Rev. A 96, 013833 (2017).

[21] J. Verdú, H. Zoubi, C. Koller, J. Majer, H. Ritsch, and J. Schmiedmayer, Phys. Rev. Lett. 103, 043603 (2009).
[22] M. Kiffner, A. Feizpour, K. T. Kaczmarek, D. Jaksch, and J. Nunn, New J. Phys. 18, 093030 (2016).

[23] J. Han, T. Vogt, C. Gross, D. Jaksch, M. Kiffner, and W. Li, Phys. Rev. Lett. 120, 093201 (2018).

[24] Y. Tabuchi, S. Ishino, T. Ishikawa, R. Yamazaki, K. Usami, and Y. Nakamura, Phys. Rev. Lett. 113, 083603 (2014).

[25] X. Zhang, C.-L. Zou, L. Jiang, and H. X. Tang, Phys. Rev. Lett. 113, 156401 (2014).

[26] L. Bai, K. Blanchette, M. Harder, Y. P. Chen, X. Fan, J. Q. Xiao, and C. M. Hu, IEEE Trans. Magn. 52, 1 (2016).

[27] M. Goryachev, W. G. Farr, D. L. Creedon, Y. Fan, M. Kostylev, and M. E. Tobar, Phys. Rev. Appl. 2, 054002 (2014).

[28] D. Marcos, M. Wubs, J. M. Taylor, R. Aguado, M. D. Lukin, and A. S. Sørensen, Phys. Rev. Lett. 105, 210501 (2010).

[29] Y. Kubo, F. R. Ong, P. Bertet, D. Vion, V. Jacques, D. Zheng, A. Dréau, J.-F. Roch, A. Auffeves, F. Jelezko, J. Wrachtrup, M. F. Barthe, P. Bergonzo, and D. Esteve, Phys. Rev. Lett. 105, 140502 (2010).

[30] L. A. Williamson, Y.-H. Chen, and J. J. Longdell, Phys. Rev. Lett. 113, 203601 (2014)..

[31] C. O’Brien, N. Lauk, S. Blum, G. Morigi, and M. Fleischhauer, Phys. Rev. Lett. 113, 063603 (2014).

[32] X. Fernandez-Gonzalvo, Y.-H. Chen, C. Yin, S. Rogge, and J. J. Longdell, Phys. Rev. A 92, 062313 (2015).

[33] X. Fernandez-Gonzalvo, S. P. Horvath, Y.-H. Chen, and J. J. Longdell, arXiv:1712.07735.

[34] M. J. Sellars, E. Fraval, and J. J. Longdell, J. Lumin. 107, 150 (2004).

[35] R. L. Ahlefeldt, M. R. Hush, and M. J. Sellars, Phys. Rev. Lett. 117, 250504 (2016).

[36] C. W. Thiel, T. Böttger, and R. L. Cone, J. Lumin. 131, 353 (2011).

[37] R. M. Macfarlane, R. S. Meltzer, and B. Z. Malkin, Phys. Rev. B 58, 5692 (1998).

[38] E. G. Spencer, R. C. LeCraw, and A. M. Clogston, Phys. Rev. Lett. 3, 32 (1959).

[39] R. Hisatomi, A. Osada, Y. Tabuchi, T. Ishikawa, A. Noguchi, R. Yamazaki, K. Usami, and Y. Nakamura, Phys. Rev. B 93, 174427 (2016).

[40] J. A. Haigh, S. Langenfeld, N. J. Lambert, J. J. Baumberg, A. J. Ramsay, A. Nunnenkamp, and A. J. Ferguson, Phys. Rev. A 92, 063845 (2015). 
[41] J. A. Haigh, N. J. Lambert, S. Sharma, Y. M. Blanter, G. E. W. Bauer, and A. J. Ramsay, Phys. Rev. B 97, 214423 (2018).

[42] J. M. Kindem, J. G. Bartholomew, P. J. T. Woodburn, T. Zhong, I. Craiciu, R. L. Cone, C. W. Thiel, and A. Faraon, Phys. Rev. B 98, 024404 (2018).

[43] A. Ortu, A. Tiranov, S. Welinski, F. Fröwis, N. Gisin, A. Ferrier, P. Goldner, and M. Afzelius, Nat. Mater. 17, 671 (2018).

[44] S. Welinski, P. J. T. Woodburn, N. Lauk, R. L. Cone, C. Simon, P. Goldner, and C. W. Thiel, Phys. Rev. Lett. 122, 247401 (2019).

[45] J. V. Rakonjac, Y.-H. Chen, S. P. Horvath, and J. J. Longdell, arXiv: 1802.03862

[46] L. R. Walker, Phys. Rev. 105, 390 (1957).

[47] P. C. Fletcher and R. O. Bell, J. Appl. Phys. 30, 687 (1959).

[48] P. Röschmann and H. Dötsch, Phys. Status Solidi (b) 82, 11 (1977).

[49] M. J. Collett and C. W. Gardiner, Phys. Rev. A 30, 1386 (1984).

[50] E. Lagendijk and W. Huiskamp, Physica 65, 118 (1973).

[51] D. L. Kepert, J. M. Patrick, and A. H. White, Aust. J. Chem. 36, 477 (1983).
[52] L. Couture and K. Rajnak, Chem. Phys. 85, 315 (1984).

[53] D. Li, M. Jiang, S. Cueff, C. M. Dodson, S. Karaveli, and R. Zia, Phys. Rev. B 89, 161409(R) (2014).

[54] See Supplemental Material at http://link.aps.org/supplemental/ 10.1103/PhysRevA.99.063830 for detailed calculations of system parameters.

[55] M. M. Abraham, J. M. Baker, B. Bleaney, J. Z. Pfeffer, and M. R. Wells, J. Phys.: Condens. Matter 4, 5443 (1992).

[56] M. I. Kaganov, in Ferromagnetic Resonance, edited by S. V. Vonsovskii (Pergamon Press, Oxford, 1966), pp. 127-143.

[57] R. M. White, Quantum Theory of Magnetism, 3rd ed. (SpringerVerlag, Berlin, 2007), Chap. 8.2.1, pp. 240-247

[58] C. Cohen-Tannoudji, J. Dupont-Roc, and G. Grynberg, Atom-Photon Interactions: Basic Process and Applications (Wiley-VCH Verlag GmbH \& Co. KGaA, Weinheim, 1992), Chap. 3.

[59] E. Brion, L. H. Pedersen, and K. Mølmer, J. Phys. A: Math. Theor. 40, 1033 (2007).

[60] T. Holstein and H. Primakoff, Phys. Rev. 58, 1098 (1940). 